\title{
LAS SIGLAS EN EL LENGUAJE ECONÓMICO DE LA PRENSA ESPAÑOLA GENERALISTA
}

\author{
Marie-Evelyne Le Poder \\ Universidad de Granada \\ lepoder@ugr.es
}

\begin{abstract}
Resumen: El presente artículo se propone llevar a cabo un trabajo de observación, análisis y descripción de las siglas, entendidas como variantes de unidades terminológicas, en el lenguaje económico de la prensa española generalista. Pretende establecer unos criterios mínimos concisos y medibles tales como su tipología, caracterización y patrones de aparición. Nuestra investigación se fundamenta en un corpus textual construido a partir de las secciones que el diario El País dedica a la información económica en un período comprendido entre agosto de 2007 y agosto de 2010. Por lo tanto, se desarrolla en el marco de una situación comunicativa de periodista (especializado) a público en general.

La propuesta metodológica se articula en torno a los enfoques comunicativos de la terminología y la lingüística de corpus. Se apoya en la terminografía basada en el uso de corpus textuales que defiende que los términos se deben observar, analizar y describir en el contexto situacional y social en el que se utilizan.
\end{abstract}

Palabras clave: Análisis, siglas, lenguaje económico, prensa española generalista, corpus textual.

\section{ACRONYMS IN ECONOMIC LANGUAGE OF THE NONSPECIALISED SPANISH PRESS}

\begin{abstract}
This article presents the observation, analysis and description of acronyms, as variants of terminological units, in the economic language of the general Spanish Press. The study aims to establish concise, measurable minimum criteria such as their typology, characteristics, and patterns of appearance.
\end{abstract}


Our research is based on a text corpus collected from the economic sections of the daily Spanish newspaper El Pais between August 2007 and August 2010. The corpus, then, is situated in a communicative situation between a journalist (specialist) and the general reading public.

Our methodology stems from from the communicative approaches of Terminology and Corpus Linguistics. It also draws on Terminography bsed on the use of textual corpora, which defends the observation, analysis and description of terms in the situational and social contexts in which they are used.

Keywords: Analysis, acronyms, economic language, popular press, textual corpus.

\section{La Teoría Comunicativa de la Terminología (TCT): teo- ría de base lingüístico-comunicativo y corte cognitivo}

A lo largo del siglo XX, los cambios sociales ocurridos han cuestionado gran parte de los valores que se habían impuesto hasta entonces y han obligado a reconsiderar aspectos de la organización social, económica y política imperantes. Algunos de estos cambios han afectado a los sistemas de comunicación, al estatus de la lengua, y al valor de la terminología especializada en la transferencia del conocimiento (Cabré 2005: 4).

Aspectos importantes han repercutido en la concepción de las vertientes teórica y práctica de la terminología. Entre ellos, las situaciones de comunicación especializada se han multiplicado, debido a la diversidad de necesidades en la transmisión del conocimiento especializado. Los contenidos especializados también se han diversificado abriéndose a las ciencias sociales y humanas, y el conocimiento experto se ha difundido a través de los medios de comunicación de masa (Ibid).

Además de estos cambios, el papel desempeñado por la lingüística en su apertura a la pragmática y la semántica, ha influido directamente en el giro cognitivo de la terminología. En este sentido, ha sido fundamental la reconsideración, por parte de la 
lingüística, del elemento léxico en sí mismo, y no sólo como componente necesario para la sintaxis. Pero todavía más decisivos han sido el nacimiento del funcionalismo lingüístico y las posteriores aportaciones cognitivas al lenguaje. Por todo ello, la teoría de la terminología se ha ido asentando y ha establecido una propuesta descriptiva de las unidades lingüísticas con la consideración de sus elementos semánticos y pragmáticos. En paralelo, la aparición de la lingüística de corpus ha sellado la evolución de la terminología hacia la vertiente descriptiva.

La reconsideración de los aspectos teóricos presentes en la teoría wüsteriana ha tenido lugar desde tres ámbitos fundamentales y se ha plasmado en la TCT que se concibe como una teoría lingüística amplia de las unidades terminológicas, de base cognitiva y propósito comunicativo (Cabré 2001: 22-25).

Desde las ciencias del lenguaje se ha denunciado la clara separación entre el lenguaje general y el especializado. En el marco de una teoría del lenguaje natural, las unidades terminológicas no son unidades que están separadas de las palabras y comparten espacio en el léxico de un hablante, sino que se conciben como valores especializados de las unidades léxicas comprendidas en el lexicón del hablante (Cabré 2005: 9). Además, una unidad léxica no se considera terminológica o general; se trata de una unidad general que logra un valor especializado cuando debido a las características pragmáticas del discurso su significado especializado se activa (Ibid).

Desde el ámbito de las ciencias cognitivas se ha hecho hincapié en la dificultad de una separación nítida entre el conocimiento especializado y el general.

Finalmente, las ciencias de la comunicación se han interesado por la diversidad de situaciones comunicativas en las que se integra la comunicación especializada. 


\section{Las siglas}

\subsection{Variantes de unidades terminológicas}

La TCT considera que una sigla es una variante de una unidad terminológica y ésta una unidad de conocimiento (Cabré et al. Giraldo 2006: 100). Por lo tanto, la sigla alcanza un estatus similar al de su forma desarrollada, y adquiere la condición de unidad terminológica.

El avance del conocimiento contribuye a que la sigla, como procedimiento de creación léxica, se utilice con frecuencia en diversas áreas del saber y aparezca en situaciones comunicativas heterogéneas. Este fenómeno lingüístico se ve también influido por factores diversos tales como la economía lingüística, la mnemotecnia, la estilística o los criterios editoriales (Ibid: 107).

\subsection{Tipología}

Una sigla es una unidad constituida por los caracteres alfanuméricos que provienen de una unidad léxica -o término- caracterizado por una estructura sintagmática. Su pronunciación puede ser de tipo alfabético, silábico o ambos.

Desde una perspectiva terminológica, se identifican tres tipos de siglas: las siglas propias, las siglas impropias y los siglónimos.

Las siglas propias se forman con las iniciales de unidades léxicas de estructura sintagmática. Ejemplo: AFI (Analistas Financieros Internacionales).

Las siglas impropias se constituyen en base a caracteres secundarios, es decir letras que no son iniciales de la unidad léxica, cifras, símbolos, o por la omisión de elementos clave de la forma desarrollada.

Estas siglas impropias se descomponen, a su vez, en tres subcategorías: siglas impropias típicas, acrónimos o cruces léxicos. 
Las denominadas siglas impropias típicas conforman unidades que utilizan o prescinden de elementos clave de su forma desarrollada y que se pronuncian de modo alfabético, silábico o ambos. Ejemplo: AOP (Asociación Española de Operadores de Productos Petrolíferos).

Los acrónimos se refieren a unidades formadas por diversos grupos de letras de sus elementos y su pronunciación es silábica. Ejemplo: MIBOR (Madrid Interbanking Offered Rate).

En cuanto a los cruces léxicos, unidades comparables al acrónimo, están constituidos por la combinación de dos segmentos de una unidad léxica con estructura sintagmática y su pronunciación es silábica. Pueden presentar distintas formas en conformidad con los segmentos que los componen y combinar los segmentos iniciales del primer y segundo elemento del sintagma. Ejemplo: ECOFIN (Economic and Financial Affairs Council).

Los siglónimos son siglas que se han integrado en la lengua general como una palabra y se han subordinado a ésta. En un primer momento las siglas se escriben en mayúsculas, pero el resultado final de la lexicalización se traduce en la pérdida de las mayúsculas. Ejemplo: I.V.A., luego IVA y, finalmente iva (Impuesto sobre el Valor Añadido).

\section{Objetivo de nuestro estudio}

El objetivo de la presente investigación es observar, analizar y describir las particularidades de las siglas en el discurso económico de divulgación. Aspiramos a establecer unos criterios mínimos concisos y medibles tales como su tipología, caracterización y patrones de aparición. La observación y descripción se llevan a cabo en el discurso natural. En este sentido, hemos trabajado a partir de las secciones que el diario El País dedica a la información económica en un período comprendido entre agosto de 2007 y agosto de 2010. 


\section{Metodología}

Nuestra propuesta metodológica se articula en torno a los enfoques comunicativos de las siguientes ramas de la lingüística aplicada: la terminología y la lingüística de corpus. Se fundamenta en la vertiente aplicada de la terminología, la terminografía basada en el uso de corpus textuales. La terminografía basada en corpus nace de la aproximación que se da entre la terminografía y la lexicografía en el contexto de las nuevas posiciones teóricas que corroboran que los términos se deben observar, analizar y describir en el contexto situacional y social en el que se utilizan y que, por ende, el estudio de su uso real es necesario. La observación y descripción se lleva a cabo a partir de los textos reales, producidos en distintas situaciones de producción y transmisión de conocimiento. En este sentido, la terminología descriptiva de índole comunicativa converge con la lingüística de corpus y recoge su metodología de análisis de corpus informatizados.

Debido al impresionante desarrollo de Internet y a la disponibilidad de publicaciones y documentos en formato electrónico, los corpus se han vuelto una herramienta imprescindible. Hoy en día, sería impensable observar, analizar y describir unidades léxicas sin utilizar un corpus textual.

Para su elaboración, hemos optado por el diario generalista $E l$ País. Este diario se caracteriza por las frecuentes contribuciones en sus páginas de distinguidos economistas y periodistas especializados que actúan como intermediarios mediáticos y se expresan en un lenguaje más inteligible para los no expertos en economía, sin por ello abandonar el rigor necesario a sus análisis y el uso de una terminología específica. El análisis de nuestro corpus es de tipo cualitativo.

Hemos aplicado al corpus la herramienta Wordsmith Tools, que ofrece un conjunto de programas para el análisis textual. 


\section{Las siglas en el lenguaje de la economía: resultados y comentarios}

\subsection{Resultados}

El trabajo de observación, análisis y descripción de las siglas de nuestro corpus que abarca 531 textos nos ha permitido comprobar:

1. El número de siglas individuales: 122

TABLA 1: Siglas por orden alfabético

\begin{tabular}{|l|l|l|l|l|}
\hline ACERINOX & CAM & FICE & ISM & VAB \\
\hline AEB & CBS & FMI & IVA & VIX \\
\hline AETIC & CCM & FROB & LIBOR & \\
\hline AFGE & CDS & FTI & LTCM & \\
\hline AFI & CECA & FTSE & LTV & \\
\hline AHE & CECU & GAMESA & $\begin{array}{l}\text { METROVA- } \\
\text { CESA }\end{array}$ & \\
\hline AICE & CEMFI & GM & MIFID & \\
\hline AIE & CEO & G7 & NRF & \\
\hline AIG & CEOE & G8 & NYSE & \\
\hline ANFAC & CEPAL & G20 & OCDE & \\
\hline ANIACAM & CEPS & HBOS & OCU & \\
\hline AOP & CEPYME & HRE & OIT & \\
\hline APCE & CERA & HSBC & OMC & \\
\hline ASNEF & CGD & IBERDROLA & ONU & \\
\hline ATTAC & CIS & IBEX & OPA & \\
\hline BAI & CNC & ICD & OPEP & \\
\hline BANESTO & CNMV & ICF & OTS & \\
\hline BBK & DAX & ICO & PIB & \\
\hline BBVA & DNB & IEE & PYMES & \\
\hline BCE & ECOFIN & IKB & RBS & \\
\hline BCG & EFR & IMFC & REIT & \\
\hline & & & & \\
\hline
\end{tabular}




\begin{tabular}{|l|l|l|l|l|}
\hline & ENDESA & INFITEX & REPSOL & \\
\hline BID & EPA & INE & RSE & \\
\hline BIS & EURIBOR & INEM & SACYR & \\
\hline BME & EUROSTAT & ING & SCIMI & \\
\hline BNP & FDIC & IPC & SFL & \\
\hline BOC & FED & IPI & SWFI & \\
\hline BP & FENOSA & IPIC & TAE & \\
\hline BPN & FGD & IREA & UBS & \\
\hline CAC & FIAT & IRPF & UE & \\
\hline TOTAL: 122 & & & & \\
\hline
\end{tabular}

2. La frecuencia de aparición de cada una de estas siglas

TABLA 2: Siglas por frecuencia de aparición

\begin{tabular}{|l|l|l|l|l|}
\hline BCE 299 & IRPF 16 & BEI 4 & FICE 2 & SCIMI 1 \\
\hline PIB 252 & OMC 16 & CEPAL 4 & FTI 2 & VAB 1 \\
\hline FMI 158 & CNMV 15 & CEPYME 4 & G8 2 & \\
\hline UE 117 & OPEP 14 & G20 4 & ICF 2 & \\
\hline IBEX 108 & ANFAC 13 & HRE 4 & IMFC 2 & \\
\hline FED 102 & CAM 12 & IPI 4 & IPIC 2 & \\
\hline BBVA 94 & HSBC 12 & IREA 4 & LTCM 2 & \\
\hline ICO 53 & AEB 1 1 & BID 3 & LTV 2 & \\
\hline OCDE 42 & CCM 10 & CEO 3 & NYSE 2 & \\
\hline ING 38 & CECA 10 & FGD 3 & OCU 2 & \\
\hline GM 37 & OPA 10 & FROB 3 & OIT 2 & \\
\hline BNP 34 & FDIC 9 & FTSE 3 & REIT 2 & \\
\hline PYMES 33 & IKB 9 & IEE 3 & SFL 2 & \\
\hline IPC 28 & RBS 9 & INDITEX 3 & SWFI 2 & \\
\hline INE 24 & APCE 8 & ISM 3 & VIX 2 & \\
\hline BANESTO 22 & DAX 8 & LIBOR 3 & AETIC 1 & \\
\hline ENDESA 22 & ONU 8 & NRF 3 & AFGE 1 & \\
\hline
\end{tabular}




\begin{tabular}{|l|l|l|l|l|}
\hline HBOS 22 & ECOFIN 7 & RSE 3 & AICE 1 & \\
\hline UBS 21 & IVA 7 & ATTAC 2 & AOP 1 & \\
\hline EUROSTAT 20 & ACERINOX 6 & BBK 2 & ASNEF 1 & \\
\hline AFI 19 & AIE 6 & BCG 2 & BAI 1 & \\
\hline $\begin{array}{l}\text { METROVACESA } \\
19\end{array}$ & INEM 6 & BME 2 & BIS 1 & \\
\hline IBERDROLA 18 & TAE 6 & BP 2 & BOC 1 & \\
\hline REPSOL 18 & BPN 5 & CBS 2 & CECU 1 & \\
\hline CEOE 17 & CAC 5 & CDS 2 & CEMFI 1 & \\
\hline SACYR 17 & GAMESA 5 & CEPS 2 & CNC 1 & \\
\hline AIG 16 & G7 5 & CERA 2 & DNB 1 & \\
\hline EPA 16 & ICD 5 & CGD 2 & EURI- & \\
\hline FENOSA 16 & AHE 4 & CIS 2 & MIFID 1 & \\
\hline FIAT 16 & ANIACAM 4 & EFR 2 & OTS 1 & \\
\hline TOTAL 2091 & & & & \\
\hline
\end{tabular}

3. La diversidad de siglas ateniéndonos a su tipología: siglas propias, siglas impropias y siglónimos

TABLA 3: Siglas según tipología (1). Siglas propias

\begin{tabular}{|l|l|l|l|}
\hline AEB & BP & FMI & LTV \\
\hline AETIC & BPN & FROB & NRF \\
\hline AFGE & CAC & FTI & NYSE \\
\hline AFI & CAM & FTSE & OCDE \\
\hline AHE & CBS & GM & OCU \\
\hline AICE & CCM & HRE & OIT \\
\hline AIE & CDS & ICD & OMC \\
\hline AIG & CECA & ICF & ONU \\
\hline ANIACAM & CEO & ICO & OPA \\
\hline APCE & CEOE & IEE & OPEP \\
\hline ATTAC & CEPS & IKB & OTS \\
\hline
\end{tabular}




\begin{tabular}{|l|l|l|l|}
\hline BAI & CERA & IMFC & PIB \\
\hline BBK & CGD & INE & RBS \\
\hline BBVA & CIS & ING & REIT \\
\hline BCE & CNC & IPC & RSE \\
\hline BCG & CNMV & IPI & SCIMI \\
\hline BEI & DNB & IPIC & SFL \\
\hline BID & EPA & IREA & SWFI \\
\hline BIS & FDIC & IRPF & TAE \\
\hline BME & FGD & ISM & UBS \\
\hline BNP & FIAT & IVA & UE \\
\hline BOC & FICE & LTCM & VAB \\
\hline TOTAL: 88 & & & \\
\hline
\end{tabular}

TABLA 4: Siglas según tipología (1). Siglas impropias

\begin{tabular}{|l|l|l|l|}
\hline ACERINOX & ECOFIN & G20 & PYMES \\
\hline ANFAC & EFR & HBOS & REPSOL \\
\hline AOP & ENDESA & HSBC & SACYR \\
\hline ASNEF & EURIBOR & IBERDROLA & VIX \\
\hline BANESTO & EUROSTAT & IBEX & \\
\hline CECU & FED & INDITEX & \\
\hline CEMFI & FENOSA & INEM & \\
\hline CEPAL & GAMESA & LIBOR & \\
\hline CEPYME & G7 & METROVACESA & \\
\hline DAX & G8 & MIFID & \\
\hline TOTAL: 34 & & & \\
\hline
\end{tabular}

TABLA 5: Siglas según tipología (1). Siglónimos

EUROSTAT

INEM

IVA 
OPA

TOTAL: 5

4. La presencia, dentro de esta tipología, de siglas nominales y siglas que actúan como apelativos. Las siglas nominales se utilizan para referirse a organizaciones internacionales, instituciones gubernamentales, sociedades bancarias y grupos financieros, sociedades bursátiles, asociaciones, confederaciones, etc. Las siglas que actúan como simples apelativos son denominaciones genéricas que clasifican la realidad y se emplean para nombrar impuestos, trabajos estadísticos, instrumentos bancarios, índices e indicadores económicos, tasas de referencia, etc. (Gómez de Enterría, 1992, p. 268).

TABLA 6: Siglas según tipología (2). Siglas nominales

\begin{tabular}{|l|l|l|l|l|}
\hline ACERINOX & BNP & EURIBOR & INE & UBS \\
\hline AEB & BOC & FED & INEM & UE \\
\hline AETIC & BP & FENOSA & ING & VAB \\
\hline AFGE & BPN & FIAT & IREA & \\
\hline AFI & CAM & FICE & ISM & \\
\hline AHE & CBS & FMI & $\begin{array}{l}\text { METROVACE- } \\
\text { SA }\end{array}$ & \\
\hline AICE & CCM & FTI & MIFID & \\
\hline AIE & CECA & FTSE & NRF & \\
\hline AIG & CECU & GAMESA & NYSE & \\
\hline ANFAC & CEMFI & GM & OCDE & \\
\hline ANIACAM & CEO & G7 & OCU & \\
\hline AOP & CEOE & G8 & OIT & \\
\hline APCE & CEPAL & G20 & OMC & \\
\hline ASNEF & CEPS & HBOS & ONU & \\
\hline ATTAC & CEPYME & HRE & OPEP & \\
\hline BANESTO & CERA & HSBC & OTS & \\
\hline
\end{tabular}




\begin{tabular}{|l|l|l|l|l|}
\hline & CGD & IBERDROLA & PYMES & \\
\hline BBV & CIS & ICD & RBS & \\
\hline BCE & CNC & ICF & REIT & \\
\hline BCG & CNMV & ICO & REPSOL & \\
\hline BEI & DNB & IEE & SACYR & \\
\hline BID & ECOFIN & IKB & SCIMI & \\
\hline BIS & EFR & IMFC & SFL & \\
\hline BME & ENDESA & INFITEX & SWFI & \\
\hline TOTAL: 99 & & & & \\
\hline
\end{tabular}

TABLA 7: Siglas según tipología (2). Siglas "apelativos"

\begin{tabular}{|l|l|}
\hline BAI & IPIC \\
\hline CAC & IRPF \\
\hline CDS & IVA \\
\hline DAX & LIBOR \\
\hline EPA & LTCM \\
\hline EURIBOR & LTV \\
\hline EUROSTAT & MIFID \\
\hline FDIC & OPA \\
\hline FGD & PIB \\
\hline FROB & RSE \\
\hline IBEX & TAE \\
\hline IPC & VAB \\
\hline IPI & VIX \\
\hline TOTAL: 26 & \\
\hline
\end{tabular}

5. La colocación de la sigla entre paréntesis en la parte derecha de su correspondiente forma desarrollada: Forma desarrollada + (sigla). Excepción: Hemos encontrado 7 veces la sigla EUROSTAT acompañada de su forma desarrollada a la derecha (...Eurostat, la oficina estadística europea...). 
6. La presencia de siglas originales en español sin su forma desarrollada: $=40,36 \%$ del total de siglas (2092).

7. La presencia de siglas en español que representan a organismos internacionales y tienen una traducción estandarizada, sin su forma desarrollada $(27,11 \%)$.

8. La presencia de siglas originales en ingles sin su forma desarrollada $(10,28 \%)$.

9. La presencia de siglas en español que representan a organismos internacionales y tienen una traducción estandarizada, acompañadas de su forma desarrollada $=5,97 \%$ del total.

10. La presencia de siglas originales en español acompañadas de su forma desarrollada $=5,26 \%$ del total.

11. La presencia de siglas originales en francés sin su forma desarrollada $=2,96 \%$ del total.

12. La presencia de siglas originales en neerlandés sin su forma desarrollada $=1,81 \%$ del total.

13. La presencia de siglas originales en ingles acompañadas de la traducción al español de su forma desarrollada. No se ha acuñado una sigla en español $=1,57 \%$ del total.

14. La presencia de siglas originales en inglés acompañadas de su forma desarrollada $=1,57 \%$ del total.

15. La presencia de siglas con combinación de segmentos en español e inglés $=0,86 \%$. del total

16. La presencia de siglas originales en alemán sin su forma desarrollada $=0,81 \%$ del total.

17. La presencia de siglas originales en italiano sin su forma desarrollada $=0,76 \%$ del total.

18. La presencia de siglas originales en portugués sin su forma desarrollada $=0,23 \%$ del total.

19. La presencia de siglas originales en portugués acompañadas de su forma desarrollada $=0,09 \%$ del total.

20. La presencia de siglas originales en neerlandés acompañadas de la traducción al español de su forma desarrollada. No se ha acuñado una sigla en español $=0,09 \%$ del total. 
21. La presencia de siglas originales en inglés acompañadas de la traducción al español de su forma desarrollada. Se ha acuñado una sigla en español, pero no se emplea $=0,09 \%$ del total.

22. La presencia de siglas no originales en español sin su forma desarrollada $=0,04 \%$ del total.

23. La presencia de siglas originales en francés acompañadas de la traducción al español de su forma desarrollada. Se ha acuñado una sigla en español, pero no se emplea $=0,04 \%$ del total.

\subsection{Ilustraciones}

5.2.1. Siglas originales en español sin su forma desarrollada $(40,36 \%)$

Siglas propias

Siglas nominales

- Sociedades bursátiles

\section{CNMV}

Un conjunto de once cajas han presentado ante la CNMV un fondo de titularización de activos de cédulas territoriales, para lo que han tenido que presentar sus resultados al cierre de 2008. (El País.com, 18 de abril de 2009).

CNMV se corresponde con Comisión Nacional del Mercado de Valores.

- Asociaciones, confederaciones

AHE

De hecho, y, según datos de la AHE, más del $78 \%$ de las familias que tienen contratado un préstamo hipotecario a tipo variable está pagando cuotas de amortización superiores a las que pagaron inicialmente. (El País.com, 19 de agosto de 2007). 
AHE es la Asociación Hipotecaria Española.

Siglas "apelativos"

- Impuestos

IVA

El sistema otorga una mayor autonomía financiera y corresponsabilidad fiscal, ya que la parte más importante de nuestro sistema tributario, como es el IVA, [...] se incrementa. (El País.com, 12 de julio de 2009).

IVA: Impuesto sobre el Valor Añadido.

- Trabajos estadísticos

$E P A$

Los datos de la EPA, el mejor termómetro del mercado laboral español, confirman las impresiones forjadas a lo largo del verano con los datos de los servicios públicos de empleo y la afiliación a la Seguridad Social. (El País.com, 27 de octubre de 2007).

EPA: Encuesta de Población Activa.

- Índices / Indicadores

IPI

Corregidos los efectos calendario, el IPI cayó un 5,5\% al compararse con mayo de 2007 (un 7,3\% sin la corrección). (El País. com, 5 de junio de 2009).

IPI: Î́ndice de Producción Industrial.

Siglas impropias

Siglas nominales

Acrónimo

\section{CEPYME}

El grifo para la deuda nueva está cerrado, afirma Carlos Ruiz, jefe del departamento económico de Cepyme, la patronal de las pequeñas firmas». (El País.com, 19 de octubre de 2008). 
CEPYME: Confederación Española de la Pequeña y Mediana Empresa.

Cruces

\section{ACERINOX}

La empresa que más factura en el extranjero es Acerinox. (El País.com, 1 de marzo de 2009).

ACERINOX: Aceros inoxidables

Siglas "apelativos"

Siglónimos

- Îndices bursátiles

IBEX: Índice Bursátil Español.

De hecho, hay ocho empresas del Ibex cuyos beneficios al cierre de año han sido inferiores de lo que lo eran a 30 de septiembre, lo que quiere decir que en los últimos tres meses contabilizaron pérdidas. (El País.com, 28 de febrero de 2009).

5.2.2. Siglas en español que representan a organismos internacionales y tienen una traducción estandarizada, sin su forma desarrollada $(27,11 \%)$.

Siglas propias

Siglas nominales

- Organizaciones internacionales

\section{FMI}

Hay varios análisis parecidos: el FMI también habló de un $20 \%$ de exceso en la red española. (El País.com, 28 de junio de 2009).

\section{$O C D E$}

Por ejemplo, el indicador de expectativas sobre la economía española que elabora la OCDE registra ligeras mejoras desde el pasado febrero tras un año ininterrumpido de deterioro. (El País. com, 12 de julio de 2009). 


\section{OPEP}

Los productores ajustan la oferta, como ha hecho la OPEP en los últimos meses. (El País.com, 7 de junio de 2009).

\section{$O M C$}

La OMC destaca en su último informe que las economías emergentes en Asia pueden frenar esa tendencia. (El País.com, 10 de abril de 2009).

5.2.3. Siglas originales en ingles sin su forma desarrollada $(10,28 \%)$.

La relevancia de los préstamos tomados de la lengua inglesa se debe a razones esencialmente económicas: es de sobra conocida la hegemonía que ejercen los países anglosajones desde el punto de vista político y económico.

Siglas propias

Siglas "apelativos"

- Índices bursátiles

FTSE

El resto de mercados del continente mostró mayor debilidad, algo que viene siendo una constante en 2008. Todos los grandes índices europeos registraron pérdidas, liderados por el FTSE. (El País .com, 21 de marzo de 2008).

FTSE o Financial Times Stock Exchange.

NYSE

En el conjunto de la semana, el Dow Jones bajó un 0,29\%, mientras que el Nasdaq subió un 0,56\%, el S\&P 500 un 0,47 por ciento y el NYSE avanzó un 1,16\%. (El País.com, 19 de septiembre de 2008).

NYSE o New York Stock Exchange se conoce como el índice de la Bolsa de Nueva York.

Siglas impropias 
Siglas nominales

- Organizaciones europeas

Siglas impropias típicas

EFR

El jueves por la noche, Durão Barroso invitó a cenar en Bruselas a los miembros del comité de dirección de la EFR, organismo formado por algunas de las entidades financieras más importantes de Europa. (El País.com, 4 de octubre de 2008).

EFR: European Financial Round Table.

La "Mesa Redonda Europea de Servicios Financieros" es la denominación que se suele utilizar para hacer referencia a la sigla inglesa EFR.

Siglas "apelativos"

- Tasas de referencia

Acrónimo

LIBOR

En segundo lugar, es necesario reanudar el préstamo interbancario ofreciendo garantías y situando el Líbor [...], en línea con los fondos de la Reserva Federal. (El País.com, 19 de octubre de 2008).

LÍBOR: London Interbank Offered Rate.

5.2.4. Siglas en español que representan a organismos internacionales y tienen una traducción estandarizada, acompañadas de su forma desarrollada $(5,97 \%)$.

Siglas propias

Siglas nominales

- Organizaciones internacionales

\section{AIE}

La situación económica más debilitada conduce a una mayor desaceleración en la demanda de crudo, resumió la OPEP, cuyos análisis coinciden con los de la Agencia Internacional de la Ener- 
gía (AIE), [...]. (El País.com, 16 de agosto de 2008).

AIE es la traducción establecida de IAE (Internacional Agency of Energy).

$B C E$

Las entidades más agobiadas, sobre todo cajas, casi han agotado las dos mangueras: el Banco Central Europeo (BCE), que presta dinero al 1,75\%, y las emisiones avalados por el Estado español. (El País.com, 14 de junio de 2009).

BCE es la traducción establecida de ECB (European Central Bank).

OIT

El último informe mundial de salarios de la Organización Internacional de Trabajo (OIT) destaca que entre 2001 y 2007 crecieron menos del 1,9\% en la mitad de los países. (El País.com, 31 de mayo de 2009).

Organización Internacional de Trabajo (OIT) es la traducción establecida para Organisation internationale du travail (OIT).

5.2.5. Siglas originales en español acompañadas de su forma desarrollada $(5,26 \%)$.

Siglas propias

Siglas nominales

- Instituciones gubernamentales

ICO

El Gobierno dio luz verde ayer al cuarto paquete de medidas anticrisis con el desarrollo, entre otras, de su medida estrella: el aplazamiento durante dos años de la mitad del pago mensual de la hipoteca a parados con cargas familiares. Y ese desarrollo implica que el Estado, a través del Instituto de Crédito Oficial (ICO), adelantará este dinero a las entidades que, voluntariamente, colabo- 
ren con la medida. (El País.com, 9 de noviembre de 2008).

- Sociedades bancarias y grupos financieros

$A E B$

Miguel Martín, presidente de la Asociación Española de Banca $(A E B)$, considera que las medidas son buenas, pero no son ayudas a la banca, sino a la economía, es decir, a las familias y a las empresas. (El País.com, 19 de octubre de 2008).

- Asociaciones, confederaciones

CNC

El presidente de la Confederación Nacional de la Construcción $(C N C)$, Juan Lazcano, afirmó ayer que lo peor de la crisis que atraviesa su sector -en referencia al desempleo- todavía está por llegar. (El País.com, 12 de julio de 2008).

Siglas "apelativos"

- Instrumentos bancarios

FGD

El diseño pactado incluye una inyección inicial de 500 millones de euros en forma de participaciones preferentes por parte del Fondo de Garantía de Depósitos (FGD), que bastaría para hacer frente a la morosidad de este año. (El País.com, 28 de marzo de 2009).

Siglas impropias

Siglas nominales

- Asociaciones, confederaciones

Acrónimo

\section{CEMFI}


El profesor Josep Pijoan-Mas, del Centro de Estudios Monetarios y Financieros (CEMFI), [...], observaba una preocupante similitud entre esta recesión y la de 1991-1994, cuando el paro trepó hasta el 24\%. (El País.com, 31 de mayo de 2009).

5.2.6. Siglas originales en francés sin su forma desarrollada $(2,96 \%)$.

Siglas propias

Siglas nominales

- Sociedades bancarias y grupos financieros BNP

Como resultado de esta transacción, el grupo Fortis abandona el negocio bancario y las actividades de seguros en Bélgica", ha explicado el consejero delegado de esta entidad, Filip Dierckx, quien ha hecho hincapié en que la integración en BNP va en el interés de todos. (El País. com, 6 de octubre de 2008).

5.2.7. Siglas originales en neerlandés inglés sin su forma desarrollada $(1,81 \%)$.

Siglas propias

Siglas nominales

- Sociedades bancarias y grupos financieros

ING

La caída bursátil de ING se une a la inestabilidad de la economía holandesa, que podría tener crecimiento cero en 2009 y 2010, según el director del Banco Holandés, Henk Brouwer. (El País. com, 18 de octubre de 2008).

5.2.8. Siglas originales en ingles acompañadas de la traducción al español de su forma desarrollada. No se ha acuñado una sigla en español $(1,57 \%)$.

Siglas propias 
Siglas nominales

- Instituciones gubernamentales

FDIC

Últimamente, la Corporación Federal de Seguros de Depósitos (FDIC) ha estado incautándose de bancos que considera insolventes a razón de aproximadamente uno por semana». (El País.com, 15 de marzo de 2009).

FDIC (Federal Deposit Insurance Corporation). Se ha acuñado la forma desarrollada Corporación Federal de Seguros de Depósitos en español.

5.2.9. Siglas originales en inglés acompañadas de su forma desarrollada $(1,57 \%)$.

Siglas propias

Siglas "apelativos"

- Instrumentos bancarios

\section{LTCM}

[...] ante la posibilidad de la quiebra de un fondo de alto riesgo, el Long Term Capital Management (LTCM), la muy liberal Reserva Federal se olvidó de sus principios de no intervención y de su filosofía de que cada palo aguante su vela [...]. (El País.com, 27 de enero de 2008).

5.2.10. Siglas con combinación de segmentos en español e inglés $(0,86 \%)$.

Siglas impropias

Siglas nominales

- Compañías

Acrónimo

REPSOL 
Eso sí, la ralentización de los ingresos ha provocado que Repsol, en un año de petróleo caro, la supere como primera por cifra de negocio. (El País.com, 28 de febrero de 2009).

5.2.11. Siglas originales en alemán sin su forma desarrollada $(0,81 \%)$.

Siglas impropias

Siglas "apelativos"

- Índices bursátiles

Acrónimo

$D A X$

El más resistente fue el DAX alemán, con una caída del 1,26\%. (El País.com, 5 de enero de 2008).

5.2.12. Siglas originales en italiano sin su forma desarrollada $(0,76 \%)$.

Siglas propias

Siglas nominales

- Compañías

\section{FIAT}

Volkswagen, por su parte, considera "muy atrevido" el alumbramiento de otro gigante europeo de la mano de Fiat, [...]. (El País.com, 7 de junio de 2009).

5.2.13. Siglas originales en portugués sin su forma desarrollada $(0,23 \%)$.

Siglas propias

Siglas nominales

- Sociedades bancarias y grupos financieros

$B P N$ 
"En ausencia de una solución que permita defender los intereses de los depositantes, el Gobierno se ha visto obligado a proponer a la Asamblea de la República la nacionalización del BPN", explicó el ministro. (El País. com, 3 de noviembre de 2008).

5.2.14. Siglas originales en portugués acompañadas de su forma desarrollada $(0,09 \%)$.

Siglas propias

Siglas nominales

- Sociedades bancarias y grupos financieros

\section{$C G D$}

"El BPN pasará a estar controlado por la Caixa Geral de Depósitos (CGD)”. (El País.com, 3 de noviembre de 2008).

5.2.15. Siglas originales en neerlandés acompañadas de la traducción al español de su forma desarrollada. No se ha acuñado una sigla en español $(0,09 \%)$.

Siglas propias

Siglas nominales

-Oficinas estadísticas

\section{CBS}

La oficina de estadística holandesa (CBS) anunció que el crecimiento en el país fue nulo (0\%). (El País.com, 16 de noviembre de 2008).

5.2.16. Siglas originales en inglés acompañadas de la traducción al español de su forma desarrollada. Se ha acuñado una sigla en español, pero no se emplea $(0,09 \%)$.

Siglas propias

Siglas nominales

- Organizaciones internacionales 
Los 185 miembros del FMI estamos comprometidos con el plan de acción del G-7, explicó en rueda de prensa Youssef BoutrosGhali, ministro de Finanzas de Egipto y presidente del Comité Monetario y Financiero Internacional (IMFC, en inglés) [...]. (El País.com, 12 de octubre de 2008).

IMFC (International Monetary and Financial Committee), se conoce como CMFI (Comité Monetario y Financiero Internacional).

- Sociedades bancarias y grupos financieros BIS

Los analistas más cualificados, como [...] el Banco de Pagos Internacionales (BIS, en sus siglas en inglés), coinciden en que este tipo de fondos acumulan activos por unos 3,3 billones de dólares. (El País.com, 14 de septiembre de 2008).

BIS (Bank for International Settlements) se conoce como BPI (Banco de Pagos Internacionales), en español.

5.2.17. Siglas no originales en español sin su forma desarrollada $(0,04 \%)$.

Siglas propias

Siglas nominales

- Sociedades bursátiles

\section{SCIMI}

Según los expertos consultados por El País, las SCIMI [...] contribuirán a introducir transparencia y profesionalizar el mercado inmobiliario. (El País.com, 19 de septiembre de 2008).

SCIMI (Sociedades Cotizadas de Inversión en el Mercado Inmobiliario) es la traducción de REIT (Real Estate Investment Trust).

5.2.18. Siglas originales en francés acompañadas de la traducción al español de su forma desarrollada $(0,04 \%)$.

Siglas propias 
Siglas nominales

- Asociaciones

AFGE

"El regreso a un enfoque a largo plazo en la definición de la estrategia por parte de los consejos de administración" debería permitir "la emergencia de una nueva gestión de empresas", afirma Jean-Aymone Massie, presidente de la Asociación Francesa de Gestión Empresarial (AFGE). (El País.com, 11 de febrero de 2008).

\subsection{Comentarios}

La primera reflexión es que la comunicación económica ya no se limita al círculo restringido de los especialistas, y su divulgación en la prensa general contribuye a que el conocimiento especializado se difunda entre un número de lectores cada vez más elevado.

Pero, ¿esta difusión del conocimiento especializado supone por parte del emisor, quien de algún modo quiere modificar el conocimiento del receptor, la consideración de las características de éste? ¿Se adapta al público general mediante procedimientos concretos que son propios del discurso de divulgación?

En nuestro trabajo hemos comprobado que la presencia de siglas originales en español sin su forma desarrollada representa el $40,36 \%$ del total; las siglas en español que representan a organismos internacionales y tienen una traducción estandarizada sin su forma desarrollada, el $27,11 \%$ del total, y las siglas originales en inglés sin su forma desarrollada, el 10,28\%.

Estos porcentajes son los que más sobresalen, seguidos por otros de menor importancia tales como el 5,97\% (siglas en español que representan a organismos internacionales y tienen una traducción estandarizada, acompañadas de su forma desarrollada), el 5,26\% (siglas originales en español acompañadas de su forma desarrollada), el 2,96\% (siglas originales en francés sin su forma desarrollada) hasta llegar a porcentajes residuales como hemos visto anteriormente. 
El hecho de que el $84,13 \%$ de las siglas de nuestro corpus se presenten sin su forma desarrollada constituye un dato que está en concordancia con el perfil sociológico del lector de El País. Respecto a la clase social, indica que casi la mitad de sus lectores/as, el $44,7 \%$, se encuadran en la clase social media-alta, el 37,9 en la alta-media, el 13,6 en la media baja, y el 3,8 en la baja-media ${ }^{1}$. En las clases sociales media-alta y alta-media se pueden incluir las siguientes categorías ocupacionales: profesionales superiores, directivos de grandes organizaciones y grandes empresarios, profesionales de nivel medio e inferior, técnicos superiores, directivos de pequeñas organizaciones, supervisores de trabajo no manual y empleadores no manuales de la administración ${ }^{2}$. Se deduce que los individuos que pertenecen a estas categorías ocupacionales gozan de niveles de formación altos o medios, por lo que su adquisición del conocimiento especializado en diversos ámbitos, tales como la economía, les permite acceder al significado de las siglas sin que sea necesario recurrir a su forma desarrollada.

\section{Conclusión}

En este trabajo de observación, análisis y descripción de las siglas, hemos comprobado la diversidad tipológica de las mismas: siglas propias, impropias, siglónimos, siglas nominales y siglas que actúan como "apelativos". Pero, también, hemos constatado que el adecuarse al nivel de competencia del receptor se refleja en el uso de procedimientos y estrategias concretas por parte del emisor. En efecto, la comunicación especializada requiere que la terminología se acomode a cada tipo discursivo, caracterizado por la información que comparten el emisor y el receptor, y por la finalidad perseguida por el texto. Ese nivel de competencia, por parte del receptor, dicta al emisor el uso de unos u otros procedimientos lingüísticos en el transcurso del texto. Así, en la gran mayoría de los casos -el $84,13 \%$-, las siglas de nuestro corpus aparecen en el discurso sin 
su forma desarrollada, es decir sin los elementos de su compleja estructura que permiten acceder al significado. El uso de tales procedimientos lingüísticos se adecua plenamente al perfil predominante de los/as lectores/as de El País que está caracterizado por individuos que pertenecen a las clases sociales media-alta y alta-media.

En nuestra próxima investigación, ya iniciada, se amplia el estudio de estas unidades terminológicas a situaciones comunicativas de experto a semi-experto y de experto a experto.

Recebido em 06/09/11

Aceito em 24/11/11

\section{Notas}

1. Estudio General de Medios (EGM).

2. Categorías ocupacionales según criterio de J. Goldthorpe. IGLESIAS DE USSEL, J. y TRINIDAD REQUENA, A. (coords.) (2005) Leer la sociedad. Una introducción a la sociología general. Madrid: Tecnos, pp. 299.

\section{Bibliografía}

BIBER, D., et al (1998). Corpus lingüística:Investigadora idioma estructura y uso, Cambridge: Cambridge Universidad Press. 
CABRÉ, M. T. (1999). La terminología: representación y comunicación. Elementos para una teoría de base comunicativa y otros artículos, Barcelona: Institut Universitari de Lingüística Aplicada.

CABRÉ, M. T. (2001). Sumario de principios que configuran la nueva propuesta teórica. En La Terminología Científico-Técnica: Reconocimiento, Análisis y Extracción de Información Formal y Semántica, M.T. Cabré, J. Feliu (eds.), 19-25, Barcelona: Institut Universitari de Lingüística Aplicada.

CABRÉ, M. T. \& GIRALDO, J. J. (2004). Las siglas en la producción de los textos especializados: hacia una propuesta de recuperación asistida mediante BwanaNet. En: Actes de GLAT, La producció de textos d'especialitat: estructura $i$ ensenyament, 305-315, Barcelona: Universitat Politécnica de Catalunya.

CABRÉ, M.T. (2005). La terminología, una disciplina en constante evolución: pasado, presente y algunos elementos de futuro. Revista Debate Terminológico riterm.net. 1, 1-14. http://www.riterm.net/revista/n_1/cabre. pdf.

CABRÉ, M. T. \& GIRALDO, J. J. (2006). Las siglas del ámbito de genoma humano: algunas consideraciones desde el punto de vista de los LSP. En Lingüística aplicada en la sociedad de la información y la comunicación J. M. Amengual, M. Salazar (eds.), 97-108, Palma de Mallorca: Universitat de les Illes Balears. http://www.upf.edu/pdi/dtf/teresa.cabre/docums/ca06aesl. pdf. El País: http:// www.elpais.com

GÓMEZ DE ENTERRÍA, J. (1992). Las siglas en el lenguaje de la economía. Revista de Filología Románica. 9, 267-274, Madrid: Universidad Complutense. http://revistas.ucm.es/fll/0212999x/articulos/RFRM9292110267A. pdf.

PÉREZ HERNÁNDEZ, C. (2002). Explotación de los córpora textuales informatizados para la creación de bases de datos terminológicas basadas en el conocimiento. Tesis Doctoral, Universidad de Málaga.

TOGNINI BONELLI, E. (2001). Corpus Linguistics at work, Amsterdam: Benjamins. 